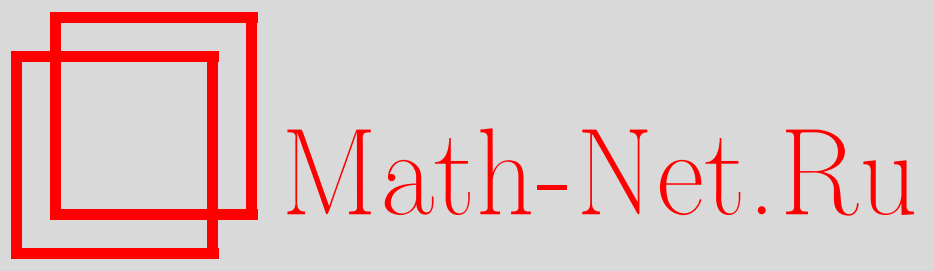

С. Ю. Славянов, Симметрии и ложные сингулярности для простейших фуксовых уравнений, ТМФ, 2017, том 193, номер 3, 401-408

DOI: https://doi.org/10.4213/tmf9382

Использование Общероссийского математического портала Math-Net.Ru подразумевает, что вы прочитали и согласны с пользовательским соглашением http://www.mathnet.ru/rus/agreement

Параметры загрузки:

IP : 52.90 .164 .192

26 апреля 2023 г., 03:57:29

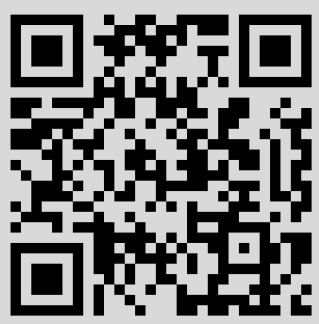




\title{
СИММЕТРИИ И ЛОЖНЫЕ СИНГУЛЯРНОСТИ ДЛЯ ПРОСТЕЙШИХ ФУКСОВЫХ УРАВНЕНИЙ
}

\begin{abstract}
Рассматриваются простейшие фуксовы уравнения второго порядка, особое внимание уделяется роли ложных сингулярностей. Показана связь с уравнением Пенлеве. Прослежена матричная формулировка проблемы.
\end{abstract}

Ключевые слова: гипергеометрическое уравнение, уравнение Гойна, деформированное уравнение Гойна, антиквантование, уравнение Пенлеве.

DOI: https://doi.org/10.4213/tmf9382

Я знал Петра Кулиша со студенческих времен. Однако мы не были близкими друзьями. Только в последнее десятилетие, когда оба мы стали членами редколлегии журнала "Теоретическая и математическая физика" и организовали конференцию по математической физике в Санкт-Петербурге, наши встречи и разговоры стали интенсивными и полезными. Он показал себя как очень грамотный и разносторонний ученый, который всегда был готов помочь окружающим людям. Его безвременная смерть явилась очень болезненным ударом для его друзей. Эта статья посвящается памяти Петра Петровича Кулиша.

\section{1. ГИПЕРГЕОМЕТРИЧЕСКОЕ УРАВНЕНИЕ}

Вначале изучается простейшее фуксово уравнение с тремя особенностями - гипергеометрическое уравнение. Это уравнение со своими конфлюэнтными формами служит базовым объектом исследования в классической теории специальных функций, которую мы здесь не собираемся представлять, а лишь подчеркнем наиболее важные, на наш взгляд, положения этой теории.

Одно возможное представление гипергеометрического уравнения таково:

$$
z(z-1) w^{\prime \prime}(z)+((a+b+1) z-c) w^{\prime}(z)+a b w(z)=0
$$

где тремя фуксовыми особенностями являются $z_{1}=0, z_{2}=1, z_{3}=\infty$. Одно из решений уравнения (1) известно как гипергеометрическая функция $F(a, b, c ; z)$. Она может быть нормирована в точке $z=0$ как $F(a, b, c ; 0)=1$. Дальнейшие

* Санкт-Петербургский государственный университет, Санкт-Петербург, Россия. E-mail: slav@ss2034.spb.edu 
обобщения уравнения (1) будут изучаться ниже. Пусть введен полиномиальный дифференциальный оператор

$$
L(D, z) w(z)=\sum_{k=0}^{2} P_{k}(z) D^{2-k} w(z)=0,
$$

где $L(D, z)$ - полином двух переменных: оператора дифференцирования $D$ и оператора умножения на комплексную переменную $z, P_{k}$ - полиномы по $z$. Полином, введенный в $(1)$, обозначим $L^{0}(D, z)$. Следовательно, $\operatorname{deg}\left(P_{k}\right)=2-k$ в (1). Пусть размерность +1 ассоциируется с умножением на $z$, а размерность -1 ассоциируется с дифференцированием $D$. Тогда $L^{0}$ имеет размерность 0 .

Наряду с уравнением (1) можно рассматривать следующую систему линейных уравнений первого порядка:

$$
R(z) \vec{Y}^{\prime}(z)=A \vec{Y}(z)
$$

Здесь $R(z)$ и $A$ - матричные полиномы с $\operatorname{deg}(R(z))=1, \operatorname{deg}(A)=0$. Можно выбрать

$$
R(z)=\left(\begin{array}{cc}
z-1 & 0 \\
1 & z
\end{array}\right), \quad A=\left(\begin{array}{cc}
-a & c+b \\
0 & -b
\end{array}\right) .
$$

Положив

$$
R^{-1} A=\frac{T}{z(z-1)}, \quad T=\left(\begin{array}{cc}
-a z & (b+c) z \\
a & -b z-c
\end{array}\right),
$$

мы придем к другой системе:

$$
z(z-1) \vec{Y}^{\prime}(z)=T(z) \vec{Y}(z) .
$$

Уравнение второго порядка для первой компоненты вектора $\vec{Y}(z)$ есть

$$
\operatorname{det}(R) y_{1}^{\prime \prime}(z)+\left[(\operatorname{det}(R))^{\prime}-\operatorname{det}(R)\left(\ln T_{12}\right)^{\prime}-\operatorname{tr}(T)\right] y_{1}^{\prime}(z)+\operatorname{det}(A) y_{1}(z)=0,
$$

что совпадает с уравнением (1).

Имеются многочисленные системы, отвечающие гипергеометрическому уравнению. Здесь выбрана система с минимальными степенями полиномов и нулевой размерности в соответствии с нашим определением.

Система (3) может быть подвергнута преобразованию Эйлера

$$
\vec{Y}(z)=\int_{l}(z-\zeta)^{\varkappa} \vec{V}(\zeta) d \zeta
$$

с подходящим выбором контура интегрирования $l$. В результате возникает следующая система для $\vec{V}(\zeta)$ вместо (3):

$$
R(\zeta) \vec{V}^{\prime}(\zeta)=(A-\varkappa I) \vec{V}(\zeta)
$$

где $I$ - единичная матрица. Сравнивая системы (3) и (5) при различных значениях $\varkappa$, мы приходим к различным симметриям гипергеометрического уравнения. В частности, может быть получено представление гипергеометрических функций через элементарные функции. 
Систему (3) можно продифференцировать, порождая уравнение для производной $\vec{U}=\vec{Y}^{\prime}$

$$
R(z) \vec{U}^{\prime}(z)=(A-I) \vec{U}(z)
$$

Суммируем результаты, полученные выше:

1) гипергеометрическое уравнение содержит три фуксовы сингулярности и характеризуется тремя параметрами, которые связаны с локальным поведением решений в окрестностях сингулярностей;

2) гипергеометрическое уравнение эквивалентно линейной системе первого порядка, которая имеет нулевую размерность;

3) дифференцирование гипергеометрического уравнения приводит к другому гипергеометрическому уравнению.

\section{2. УРАВНЕНИЕ ГОЙНА}

Следующим по сложности является фуксово уравнение, называемое уравнением Гойна с тремя фуксовыми сингулярностями в конечных точках $z_{j}, j=1,2,3$, и фуксовой точкой на бесконечности $z_{4}$. Без потери общности положим $z_{1}=0, z_{2}=1$, $z_{3}=t$. Уравнение Гойна записывается как

$$
L^{1}(D, z) w(z)=P_{0}(z) w^{\prime \prime}(z)+P_{1}(z) w^{\prime}(z)+P_{2}(z) w(z)=0
$$

где

$$
\begin{gathered}
P_{0}(z)=\prod_{j=1}^{3}\left(z-z_{j}\right)=\sigma(z), \quad P_{1}(z)=\sum_{k=1}^{3} \frac{\left(1-\theta_{k}\right) P_{0}(z)}{z-z_{k}}=\tau(z), \\
P_{2}(z)=\alpha \beta z-t(t-1) h=\omega(z)-t(t-1) h .
\end{gathered}
$$

Следовательно, $P_{0}$ является полиномом третьей степени по $z, P_{1}-$ полиномом второй степени по $z, P_{2}$ - полиномом первой степени по $z$. Как следствие дифференциальный оператор $L^{1}$ в соответствии с $(2)$ имеет размерность +1 . Для характеристических показателей в сингулярностях должно выполняться условие Фукса

$$
\sum_{k=1}^{3} \theta_{k}+\beta+\alpha=2 .
$$

Обобщенный символ Римана (ср. с [1]) для уравнения (6) имеет вид

$$
\left(\begin{array}{ccccc}
z_{1} & z_{2} & z_{3} & \infty & z \\
0 & 0 & 0 & \alpha & h \\
\theta_{1} & \theta_{2} & \theta_{3} & \beta &
\end{array}\right)
$$

Параметр $h$ называют акцессорным параметром. Выбор множителя перед ним, а именно $t(t-1)$, приводит к выполнению следующей леммы.

ЛЕмма 1. Уравнение (6) редуиируется при $t=0 u t=1 \kappa$ гипергеометрическому уравнению (1). 
Лемма 1 доказывается прямыми вычислениями. Положим, например, $t=0$ в (6). Вместо (6) получим

$$
z L^{0}(D, z)=0
$$

В противном случае параметр $h$ сохраняется в предельном уравнении. Точно так же исследуется случай $t=1$.

Интерес к уравнению Гойна и к уравнениям, генерируемым уравнением Гойна процессом конфлюэнции, растет в последние десятилетия [1], [2]. Во-первых, они более общие по сравнению с уравнениями гипергеометрического типа, а во-вторых, возникает все большее число их приложений.

Продифференцируем уравнение (6) по $z$, обозначая $u(z)=w^{\prime}(z)$. Тогда возникнет следующее уравнение:

$$
\begin{aligned}
P_{0}(z) u^{\prime \prime}(z) & +\left(P_{1}(z)+P_{0}^{\prime}(z)-P_{0}(z) \frac{1}{z-q}\right) u^{\prime}(z)+ \\
& +\left(P_{2}(z)+P_{1}^{\prime}(z)-P_{1}(z) \frac{1}{z-q}\right) u(z)=0,
\end{aligned}
$$

где $q=t(t-1) h / \alpha \beta$. Фуксова сингулярность $z=q$ оказывается одновременно так называемой ложной сингулярностью. В ложной сингулярности общее решение уравнения не содержит логарифмических членов и является голоморфной функцией для выбранного представления уравнения [3], [4]. Расположение ложной сингулярности в (7) зафиксировано. Можно рассмотреть ложную сингулярность с произвольным расположением $z=q$, а именно уравнение

$$
\begin{aligned}
\sigma(z) w^{\prime \prime}(z) & +\left(\tau(z)-\frac{\sigma(z)}{z-q}\right) w^{\prime}(z)(\omega(z)-t(t-1) h) w(z)- \\
& -\left(\sigma(q) \mu^{2}+\left(\tau(q)-\frac{\sigma(q)}{z-q}\right) \mu-t(t-1) h\right) w=0 .
\end{aligned}
$$

Мы называем уравнение (8) деформированным уравнением Гойна.

Могут быть выбраны два независимых решения $w_{1}(z)$ и $w_{2}(z)$ в $z=q$, удовлетворяющие следующим условиям: одно решение $w_{1}(z)$,

$$
w_{1}(q)=1, \quad w_{1}^{\prime}(q)=\mu, \quad w_{1}^{\prime \prime}(q)=0,
$$

и второе решение $w_{2}(z)$,

$$
w_{2}(q)=0, \quad w_{2}^{\prime}(q)=0, \quad w_{2}^{\prime \prime}(q)=1 .
$$

Введем два полинома. Первый полином

$$
H(p, z)=\frac{1}{t(t-1)}\left(\sigma(z) p^{2}+\tau(z) p+\omega(z)\right),
$$

где $p$ - квантовый оператор дифференцирования $p=d / d z$, а $z$ - оператор умножения. Это операторный полином, и мы называем его квантовым гамильтонианом. Другой полином $H(\mu, q)$,

$$
H(\mu, q)=\frac{1}{t(t-1)}\left(\sigma(q) \mu^{2}+\tau(q) \mu+\omega(q)\right),
$$


мы назовем классическим гамильтонианом от двух классических переменных: импульса $\mu$ и координаты $q$. Оба гамильтониана (9) и (10) включают $t$ как параметр, который далее ассоциируется с временем. Важно подчеркнуть, что полиномы (9) и (10) - одинаковые функции, но записанные в различных переменных.

В предложенных обозначениях уравнение (8) может быть записано в симметричной форме:

$$
\begin{aligned}
(H(p, z)-h) w(z) & -(H(\mu, q)-h) w(z)+ \\
& +\frac{1}{(z-q) t(t-1)}\left(\sigma(q) \mu w(z)-\sigma(z) w^{\prime}(z)\right)=0 .
\end{aligned}
$$

Лемма 2. Выберем в уравнении (11) решение $w_{1}$. Тогда вторая строчка в (11) пропадает при $z=q, \ln w^{\prime}(q)=\mu$, уравнение (11) обращается в тождество.

Доказательство леммы 2 основано на рассмотрении разложения Тейлора всех членов во второй строчке в (11) по степеням $z-q$ в точке $z=q$.

Теперь мы имеем время $t$ как параметр в обоих гамильтонианах. В квантовой механике варьирование времени может соответствовать адиабатической деформации квантовой системы. В случае классического гамильтониана мы можем говорить о классическом динамическом движении. Антиквантование уравнения (11) состоит из подстановки $p$ вместо $\mu$ и $z$ вместо $q$. Оно переводит уравнение (11) в тождество при дополнительном условии, что рассматривается решение $w_{1}(z)$. Ясно, что динамическое движение $\mu(t), q(t)$, определяемое гамильтонианом (10), следует либо согласно гамильтоновой системе уравнений, либо согласно уравнению Эйлера-Лагранжа.

Теорема 1. Антиквантование уравнения Гойна приводит его $к$ интегрируемому уравнению Пенлеве $P^{\mathrm{VI}}$.

Доказательство теоремы 1 основано на выводе уравнения Эйлера-Лагранжа из гамильтониана (10) и последующего сравнения с уравнением $P^{\mathrm{VI}}$.

Приведенные выше рассмотрения являются существенным развитием идей, представленных в статьях [5], [6]. Лемма 1 является новой. Лемма 2 основана на исследованиях в работах [4], [7].

Традиционное изучение деформированного уравнения Гойна развивается в терминах $(2 \times 2)$-системы линейных уравнений. Воспроизведем здесь выкладки из статьи [8], значительно изменив как изложение базовых идей, так и технику вычислений.

Выберем систему уравнений в виде

$$
R \vec{Y}^{\prime}=\left(\begin{array}{cc}
(z-t)(z-\rho) & -z+1 \\
\rho(z-t) & z-1
\end{array}\right) \vec{Y}^{\prime}=A \vec{Y}=\left(\begin{array}{cc}
\alpha z & e_{2} \\
t e_{3} & \beta
\end{array}\right) \vec{Y}
$$

Регулярные особые точки этой системы в конечной плоскости $z$ суть $z_{1}=0, z_{2}=1$, $z_{3}=t$. Присутствие параметра $\rho$ обеспечивает требуемое число параметров в результирующем уравнении второго порядка. Система (12) приводится к виду

$$
\sigma(z) \overrightarrow{Y^{\prime}}=T \vec{Y}
$$


где

$$
T(z)=\left(\begin{array}{cc}
(z-1)\left(\alpha z+t e_{3}\right) & (z-1)\left(e_{2}+\beta\right) \\
(z-t)\left(z\left(\rho \alpha+t e_{3}\right)-\rho t e_{3}\right) & (z-t)\left(\beta z-\rho\left(\beta+e_{2}\right)\right)
\end{array}\right) .
$$

Разрешая систему (13) относительно $y_{1}(z)$, приходим к уравнению второго порядка

$$
\sigma(z) y_{1}^{\prime \prime}(z)+P(z) y^{\prime}(z)+Q(z) y_{1}(z)=0
$$

где

$$
P(z)=-\sigma(z)\left(\ln \frac{T_{12}}{\sigma}\right)^{\prime}-\operatorname{tr} T, \quad Q(z)=T_{12}\left(\frac{T_{11}}{T_{12}}\right)^{\prime}+\sigma^{-1} \operatorname{det} T .
$$

Подставив выражения для матричных элементов матрицы $T$, найдем

$$
\begin{gathered}
\sigma^{-1} \operatorname{det} T=\operatorname{det} A=\alpha \beta z-t e_{2} e_{3}, \\
\operatorname{tr} T=\left(\alpha+e_{3}\right) z(z-1)+\left(\beta-\rho\left(\beta+e_{2}\right)\right) z(z-t)+\left(\rho\left(\beta+e_{2}\right)-e_{3}\right)(z-t)(z-1) .
\end{gathered}
$$

Окончательно получим

$$
\begin{gathered}
P(z)=-\left(\alpha+e_{3}\right) z(z-1)+\left(1-\beta+\rho\left(\beta+e_{2}\right)\right) z(z-t)+ \\
+\left(1+e_{3}-\rho\left(\beta+e_{2}\right)\right)(z-1)(z-t), \\
Q(z)=\alpha(\beta-1) z-t e_{2} e_{3}+\alpha .
\end{gathered}
$$

Видно, что построенное уравнение (14) совпадает с уравнением Гойна (6) при установлении правильной связи между параметрами. В уравнении (6) параметрами являются, например, $\theta_{2}, \theta_{3}, \alpha, \beta, h$, а в уравнении (14) параметрами являются $e_{2}, e_{3}$, $\rho, \alpha, \beta$. Укажем связи между параметрами:

$$
t(t-1) h=t e_{2} e_{3}-\alpha, \quad \theta_{3}=1+\alpha+e_{3}, \quad \theta_{2}=\beta-\rho\left(\beta+e_{2}\right) .
$$

Видно, что параметр $\rho$ в системе (12) тесно связан с энергией $h$.

Можно получить и уравнение второго порядка для компоненты $y_{2}(z)$. Оно является деформированным уравнением Гойна.

Системы уравненй первого порядка удобно использовать для вывода различных интегральных соотношений. Таким образом, многие базовые свойства гипергеометрических уравнений переносятся и на уравнение Гойна. То же самое наблюдается и для уравнений, возникающих при конфлюэнции фуксовых сингулярностей.

\section{3. УРАВНЕНИЕ ПЕНЛЕВЕ}

Уравнение Пенлеве - это нелинейное интегрируемое уравнение, широко изучаемое и применяемое в последние десятилетия. Недавние исследования в этой области можно найти, например, в сборнике статей [9]. Наши интересы лежали в установлении взаимно однозначного соответствия между уравнением Гойна и уравнением Пенлеве [1], [6].

Предложенный в данной статье подход служит оправданием эвристического антиквантования уравнения Гойна, предложенного в предыдущих статьях, начиная с публикации [10]. 
Мы вкратце повторим вывод уравнения $P^{\mathrm{VI}}$. Преобразование гамильтониана в лагранжиан состоит в переходе от переменной $\mu$ к переменной $\dot{q}$ и переходе от гамильтониана $H(\mu, q)$ к лагранжиану $\mathcal{L}(\dot{q}, q)$ согласно

$$
\begin{aligned}
\dot{q} & =\frac{\partial H}{\partial \mu}=\frac{2 \sigma(q) \mu+\tau(q)}{t(t-1)} \\
\mathcal{L}(\dot{q}, q) & =\dot{q} \mu-H(\mu, q)=\frac{\left((t(t-1))^{1 / 2} \dot{q}-(t(t-1))^{-1 / 2} \tau(q)\right)^{2}}{4 \sigma(q)}-\frac{\omega(q)}{t(t-1)} .
\end{aligned}
$$

Соответствующее (15) уравнение Эйлера-Лагранжа

$$
\frac{d}{d t} \frac{\partial \mathcal{L}}{\partial \dot{q}}-\frac{\partial \mathcal{L}}{\partial q}=0
$$

в изучаемом случае имеет вид

$$
\begin{aligned}
\ddot{q}-\frac{1}{2} \frac{\partial \ln \sigma(q)}{\partial q} \dot{q}^{2} & +\frac{\partial \ln (t(t-1) / \sigma(q))}{\partial t} \dot{q}- \\
& -\frac{\sigma(q)}{t^{2}(t-1)^{2}}\left[\frac{\partial}{\partial q} \frac{\tau(q)^{2}}{2 \sigma(q)}+\frac{\partial}{\partial t} \frac{\tau(q)}{\sigma(q)}-2 \frac{\partial(\omega(q))}{\partial q}\right]=0 .
\end{aligned}
$$

Прямые, хотя и трудоемкие, вычисления ведут от (16) к нелинейному уравнению, которое оказывается уравнением Пенлеве $P^{\mathrm{VI}}$ :

$$
\begin{aligned}
\ddot{q}-\frac{1}{2}\left(\frac{1}{q}+\frac{1}{q-1}+\frac{1}{q-t}\right) \dot{q}^{2}+\dot{q}\left(\frac{1}{t}+\frac{1}{t-1}+\frac{1}{q-t}\right)+ \\
+\frac{\sigma}{2 t^{2}(t-1)^{2}}\left[\frac{\left(1-\theta_{1}\right)^{2} t}{q^{2}}-\frac{\left(1-\theta_{2}\right)^{2}(t-1)}{(q-1)^{2}}+\right. \\
\left.+\frac{\left(\left(2-\theta_{3}\right)^{2}-1\right) t(t-1)}{(q-t)^{2}}-(\alpha-\beta)^{2}\right]=0 .
\end{aligned}
$$

Сравнение с уравнением Гойна (6) порождает многие размышления. Если мы вспомним, что $1-\theta_{j}, j=1,2,3$, а $\alpha, \beta$ - локальные характеристики решений уравнения Гойна в точках $z_{j}$, удовлетворяющие условию Фукса, мы немедленно получим, что $\left(1-\theta_{j}\right)^{2},(\alpha-\beta)^{2}$ являются локальными характеристиками уравнения Пенлеве $P^{\mathrm{VI}}$. Уравнение Гойна может быть подвергнуто дробно-линейному преобразованию независимой переменной и s-гомотопному преобразованию (ср. [1]) зависимой переменной. В обоих случаях свойство интегрируемости сохраняется. Более того, параметры в уравнении (17) являются инвариантами уравнения Гойна. Следовательно, различия в конечном уравнении Пенлеве будут минимальными.

Во многих случаях специальных значений параметров в уравнении (6) появляются упрощенные решения. Это дает подсказку для поиска упрощенных решений уравнения (17). Выбирая точки $z_{1}=-1, z_{2}=1$ вместо точек $z_{1}=0, z_{2}=1$ и взяв $\theta_{1}=\theta_{2}$ в уравнении (6), получаем новые симметрии уравнения (17).

Ключевая роль дополнительных ложных особенностей делает теорию уравнения Гойна отличной от теории гипергеометрического уравнения. Технические средства остаются частично теми же, но идеология является значительно более продвинутой. 
Что происходит далее при введении новых сингулярностей в фуксово уравнение? Одна добавленная сингулярность приводит к появлению второго акцессорного параметра и одной дополнительной ложной сингулярности при дифференцировании [4]. Нельзя говорить о двух гамильтонианах, и в результате свойство интегрируемости становится неверным. Открытым остается вопрос о линейных дифференциальных операторах высшего порядка, если они могут быть факторизованы.

Благодарности. Автор благодарен соавторам своих прежних публикаций в этой области за стимулирующие обсуждения, а также весьма обязан проф. М. Берри, который пригласил автора на симпозиум в Институт математики Исаака Ньютона, что обогатило его знания об уравнениях Пенлеве.

\section{Список литературы}

[1] С. Славянов, В. Лай, Специалънъе функции. Единая теория, основанная на анализе сингулярностей, Невский диалект, СПб., 2002.

[2] A. Ronveau (ed.), Heun's Differential Equation, Oxford Univ. Press, Oxford, 1995.

[3] A. V. Shanin, R. V. Craster, "Removing false singular points as a method of solving ordinary differential equations", European J. Appl. Math., 13:6 (2002), 617-639.

[4] С. Ю. Славянов, Д. А. Шатько, А. М. Ишханян, Т. А. Ротинян, "Генерация и удаление ложных особенностей в линейных обыкновенных дифференциальных уравнениях с полиномиальными коэффициентами", ТМФ, 189:3 (2016), 371-379.

[5] С. Ю. Славянов, "О понижении полиномиальной степени фуксовой $(2 \times 2)$-системы”, $T M \Phi, \mathbf{1 8 2 : 2}$ (2015), 223-230.

[6] С. Ю. Славянов, О. Л. Стесик, “Антиквантование деформированных уравнений класса Гойна", ТМФ, 186:1 (2016), 142-151.

[7] A. Ishkhanyan, K. Suominen, "New solutions of Heun's general equation", J. Phys. A: Math. Gen., 36:5 (2003), L81-L86.

[8] А.Я. Казаков, С. Ю. Славянов, "Интегральные симметрии Эйлера для деформированного уравнения Гойна и симметрии уравнения Пенлеве PVI”, TMФ, 155:2 (2008), 252-264.

[9] A.D. Bruno, A.B. Batkhin (eds.), Painlevé Equations and Related Topics, Proceedings of the International Conference (Saint Petersburg, June 17-23, 2011), De Gruyter, Berlin, 2012.

[10] S. Yu. Slavyanov, "Painlevé equations as classical analogues of Heun equations", J. Phys. A: Math. Gen., 29:22 (1996), 7329-7335. 